\title{
Effects of the Rotigotine and Venlafaxine on Sexual Dysfunction and Depression in Rats
}

\author{
Wang WQ, Meng XY, Fan YQ, Yue SM, Yang YQ, Wang XY, Wang T, Fu FH*
}

Key Laboratory of Molecular Pharmacology and Drug Evaluation, School of Pharmacy, Ministry of Education, Yantai University, Yantai, Shandong 264005, PR China

\begin{abstract}
Introduction: Rationale Venlafaxine is a serotonin-norepinephrine reuptake inhibitor (SNRI), differing from the selective serotonin reuptake inhibitors (SSRIs) in that it inhibits the reuptake of both serotonin and norepinephrine. Reportedly, the rates of sexual dysfunction with venlafaxine are lower or similar to those of the SSRIs. Dopamine plays a role in sexual behavior and is related to ejaculation.

Objectives: To assess changes in the potential effects of dopamine receptor agonist rotigotine on venlafaxine -induced sexual dysfunction and depression in rats.

Methods: The depressive-like rats models induced by olfactory bulbectomy (OB) were intragastrically administered with venlafaxine $(40 \mathrm{mg} / \mathrm{kg}$ ) and co-administered with rotigotine or rotigotine-loaded microspheres (RoMS) at dose of $20 \mathrm{mg} / \mathrm{kg}$. The sex function and anti-depressive effects were observed by copulatory behavior test (CBT) and open field test (OFT) respectively.

Result: The olfactory bulbectomy induced sexual dysfunction which significantly deteriorated by venlafaxine administration. Rotigotine could not attenuate the venlafaxine-induced sexual dysfunction, but RoMS could do it partly. The venlafaxine showed anti-depressive efficacy of OB, but the RoMS could not do better.

Conclusion: RoMS partly reverses the venlafaxine-induced sexual dysfunction. It may be better that patients with depression are co-administered with venlafaxine and RoMS.
\end{abstract}

Keywords: Depression; Venlafaxine; Rotigotine-loaded microspheres; Sexual function

\section{INTRODUCTION}

Major depressive disorder (MDD) is one of the most common and debilitating mental disorders [1]. MDD occurs frequently and causes a heavy burden on society [2]. Mood disorders are recurrent, life threatening (due to the risk for suicide), and a major cause of morbidity worldwide [3].The World Health Organization (WHO) has projected that MDD will be the second leading cause of disability by the year 2020 [4]

Sexual dysfunction is one of the serious side effects of commonly used antidepressant (AD) treatments. Among them, selective serotonin reuptake inhibitors (SSRIs) have become the most frequently prescribed drugs. SSRIs have been reported to reduce libido, delay ejaculation or orgasm, and inhibit ejaculation in humans [5]. With the advent of serotonin-norepinephrine reuptake inhibitor (SNRI), there was hope that depression could be treated with a lower incidence of SD [6]. In clinical research, which adopted a more proactive approach by asking patients direct questions about their sexual functioning, the estimated incidence of SD rose to levels ranging from $30 \%$ to $70 \%$ [7].

The mechanisms of SNRIs-induced sexual disturbances are largely inferential, derived in part from in vitro models. High central serotonin levels are associated with inhibition of libido, orgasm, and ejaculation [8]. This may result from serotonin- induced attenuation in central levels of dopamine, a neurotransmitter that is believed to mediate sexual desire and satisfaction [9]. Specifically in men, $\alpha 1$-adrenergic activity promotes venous return from the corpus cavemosa in the penis, whereas peripheral cholinergic transmission may assist in filling these penile tissues [10]. Agents with anticholinergic or $\alpha 1$-blocking properties can adversely influence arousal [11].

The other monoamine system strongly innervated by serotonin is the dopamine system. In general, DA neurotransmission has been associated with facilitation of sexual behaviour [12]. Antidepressants with a non-serotonergic mechanism of action, like

Correspondence to: Fu FH, Key Laboratory of Molecular Pharmacology and Drug Evaluation, School of Pharmacy, Yantai University, Shandong 264005 , PR China, Tel: +(086)-535-6706066; E-mail: fufenghua@sohu.com

Received: March 02, 2020; Accepted: March 15, 2020; Published: March 20, 2020

Citation: Wang WQ, Meng XY, Fan YQ, Yue SM, Yang YQ, Fu FH, et al. (2020) Effects of the rotigotine and venlafaxine on sexual dysfunction and depression in rats. J Clin Exp Pharmacol 10:264. doi: 10.35248/2161-1459.20.10.265

Copyright: (c) 2020 Wang WQ, et al. This is an open-access article distributed under the terms of the Creative Commons Attribution License, which permits unrestricted use, distribution, and reproduction in any medium, provided the original author and source are credited. 
the dopamine-noradrenaline reuptake inhibitor bupropion, have few sexual side effects, and may favour sexual function [13]. DA in mesolimbic areas is very important for the motivational and copulatory aspects of sexual behaviour. Decreasing mesolimbic DA activity delays the onset of copulation and decreases the rate of copulation and increasing mesolimbic DA facilitates computation $[14,15]$.

Rotigotine is a non-ergotamine D3/D2/D1 dopamine receptor agonist for the treatment of Parkinson's disease (PD). Rotigotineloaded microspheres (RoMS) were prepared with poly (lactide-coglycolide) as a drug carrier to achieve a long-acting sustained release formulation of rotigotine [16]. According to in vivo studies, after intramuscular (IM) administration, RoMS displayed continuousrelease characteristics of rotigotine and a steady efficacy for about 2 weeks in normal rats or PD rats induced by 6-hydroxydopamine[17].

The aim of this study was to evaluate the potential effects of the DA receptor agonist rotigotine, especially rotigotine-loaded microspheres on both sexual dysfunction and depression in bilateral olfactory bulbectomy surgery $(\mathrm{OB})$ animal models.

\section{MATERIALS AND METHODS}

\section{Animals}

The regular inspections of animal housing and the well-being of the animals were carried by licensed veterinarian. We do our best to minimize animal suffering and to reduce the number of animals used. All procedures were approved by the Yantai University Committee on the Use and Care of Animals. Sprague-Dawley rats (Jinan Pengyue company, China) weighing 200-250 g on arrival were housed individually on a reverse 12 -h light/12-h dark cycle (lights on at 20:00) in a climate-controlled room. Male and female animals were housed in different cages of the same room.

\section{Bilateral olfactory bulbectomy surgery}

Bilateral olfactory bulbectomy surgery $(\mathrm{OB})$ was performed in rats anesthetized with pentobarbital sodium $(50 \mathrm{mg} / \mathrm{kg})$. Following exposure of the skull, holes were drilled $7 \mathrm{~mm}$ anterior to bregma and $2 \mathrm{~mm}$ on either side of the midline at a point corresponding to the posterior margin of the orbit of the eye. The olfactory bulbs were removed by suction, and the burr holes were filled with a hemostatic sponge. The sham operated animals were treated similarly, but the bulbs were left intact. The skin was then closed. The rats were given 14 days to recover following surgery, and they were handled daily by the experimenter throughout the recovery period to prevent stress and/or aggressive behaviour [18].

\section{Ovariectomy surgery}

Rats were anesthetized with pentobarbital sodium $(50 \mathrm{mg} / \mathrm{kg})$ and ovaries were exposed via bilateral flank incisions and excised. All rats were administered the Penicillin (8000 U, i.p.) for up to $24 \mathrm{~h}$ after surgery and were allowed to recover for 14 days.

\section{Experimental design}

Rats were randomly assigned to 5 groups: control group, model group, venlafaxine group ( $40 \mathrm{mg} / \mathrm{kg}$ ), a venlafaxine plus RoMS (20 $\mathrm{mg} / \mathrm{kg}$ ) group and a venlafaxine plus rotigotine $(20 \mathrm{mg} / \mathrm{kg})$ group.
The rats were intragastrically administered venlafaxine which was dissolved in distilled water at dose of $40 \mathrm{mg} / \mathrm{kg}$, once a day for 7 days. At the seventh day we done the copulatory behavior test (CBT) and open field test.

\section{Copulatory behavior test}

All ovariectomized females were injected with estradiol benzoate (Sigma, MO, USA: $50 \mu \mathrm{g}$ in $0.1 \mathrm{ml}$ sesame oil) $48 \mathrm{~h}$ before the tests and progesterone (Sigma, MO, USA: $5 \mathrm{mg}$ in $0.1 \mathrm{ml}$ sesame oil) $4 \mathrm{~h}$ before the tests. Starting at 7 days after the beginning of drug administration. A pair composed of an experimental male and a stimulus female was placed in an observation cage $(52 \mathrm{~cm} \times 32 \mathrm{~cm} \times$ $19 \mathrm{~cm}$ ) and video recorded for $30 \mathrm{~min}$. This analysis determined the (1) intromission frequency, number of mounts with intromission in 30 minutes. (2) ejaculation frequency, number of ejaculations in 30 minutes. (3) mount latency, elapsed time between introduction of the female and the first display of mounting. (4) intromission latency, elapsed time between introduction of the female and the first display of intromission. (5) ejaculation latency, elapsed time between introduction of the female and the first display of ejaculation [19].

\section{Open field testing}

After 7 days of drug administration, all experimental males were tested for open-field behaviour. The test was started by placing each male in a square open-field arena $(75 \mathrm{~cm} \times 75 \mathrm{~cm})$ with 40 $\mathrm{cm}$ walls. Behaviour was video recorded for $5 \mathrm{~min}$ using a digital video camera and male locomotive behaviour was analysed off-line using commercial software (SMART3.0 software (Panlab Harvard Apparatus) [20].

\section{RESULTS}

\section{Effect of venlafaxine and RoMS on number of intromission and number of ejaculation}

In the group $\mathrm{OB}$, the number of intromission and ejaculation were significantly fewer than those in the group control $(p<0.01)$. The number of intromission and ejaculation of group venlafaxine significantly decreased compared with the rats in group $\mathrm{OB}$ $(p<0.01)$. The number of intromission and ejaculation of rats from venlafaxine group was increased by RoMS group ( $p<0.01$ ), but not changed by the rotigotine (Figure 1).

\section{Effect of RoMS on mount latency, intromission latency and ejaculation latency}

When compared with the group control the latency periods for mount, intromission and ejaculation significantly increased in the group $\mathrm{OB}(\mathrm{p}<0.05)$. The latency periods for mount, intromission and ejaculation of the rats in the venlafaxine group were higher than those in OB group $(\mathrm{p}<0.05)$. RoMS made the venlafaxinecaused latency periods for mount, intromission and the ejaculation lower $(\mathrm{p}<0.01)$. However the rotigotine could not do it like RoMS (Figure 2).

\section{Effect of RoMS on total distance and mean speed}

The total distance and mean speed of the rats in the OB group significantly increased, when compared with the control group 
rats $(\mathrm{p}<0.05)$. The mean speed and total distance was decreased in the venlafaxine group, venlafaxine with RoMS group, and venlafaxine with rotigotine group, compared with the $\mathrm{OB}$ group
( $\mathrm{p}$ <0.05). However co-administered venlafaxine with RoMS or with rotigotine did not significantly change the total distance and mean speed, compared with administered venlafaxine alone (Figure 3). a

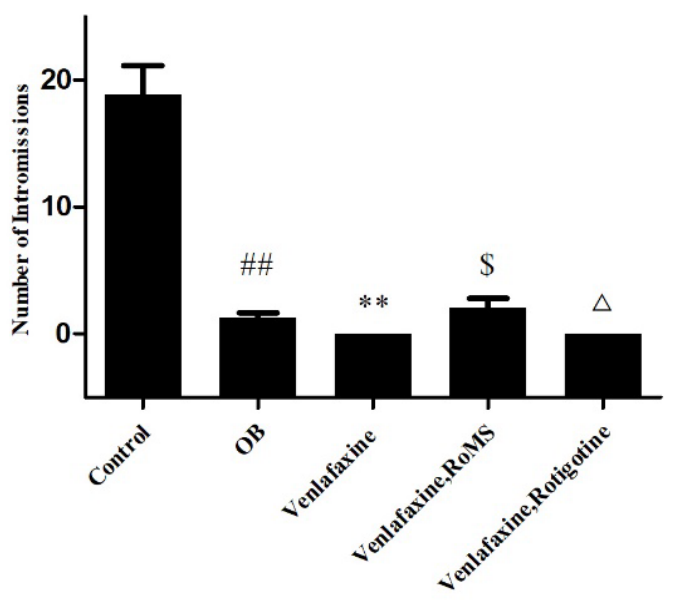

b

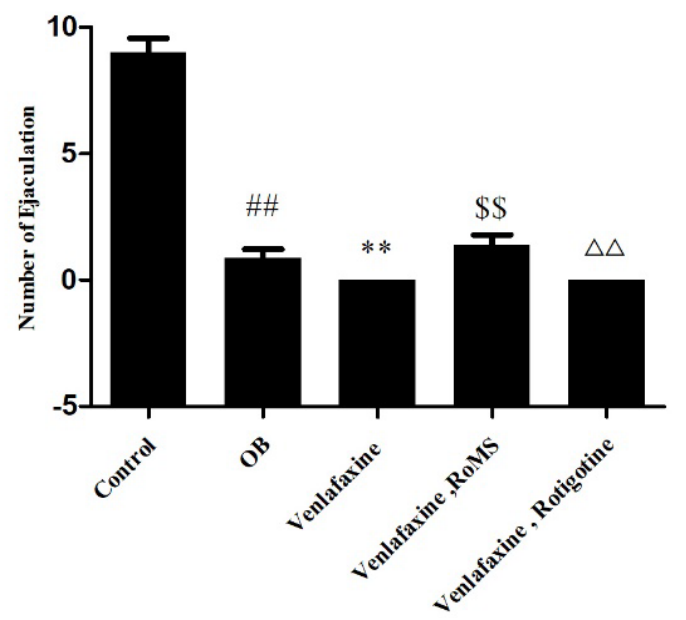

Figure 1: Effects of RoMS on (a) number of intromission and (b) number of ejaculation. Data are expressed as mean \pm SEM. ${ }^{\# \#} p<0.01$ when compared to the control group. " ${ }^{* *}<0.01$ when compared to the OB group. ${ }^{\$} p<0.05 ;{ }^{\$ \$} p<0.01$ when compared to the venlafaxine group. ${ }^{\Delta} p<0.05 ;{ }^{\Delta \Delta}<<0.01$ when compared to the venlafaxine, RoMS group.
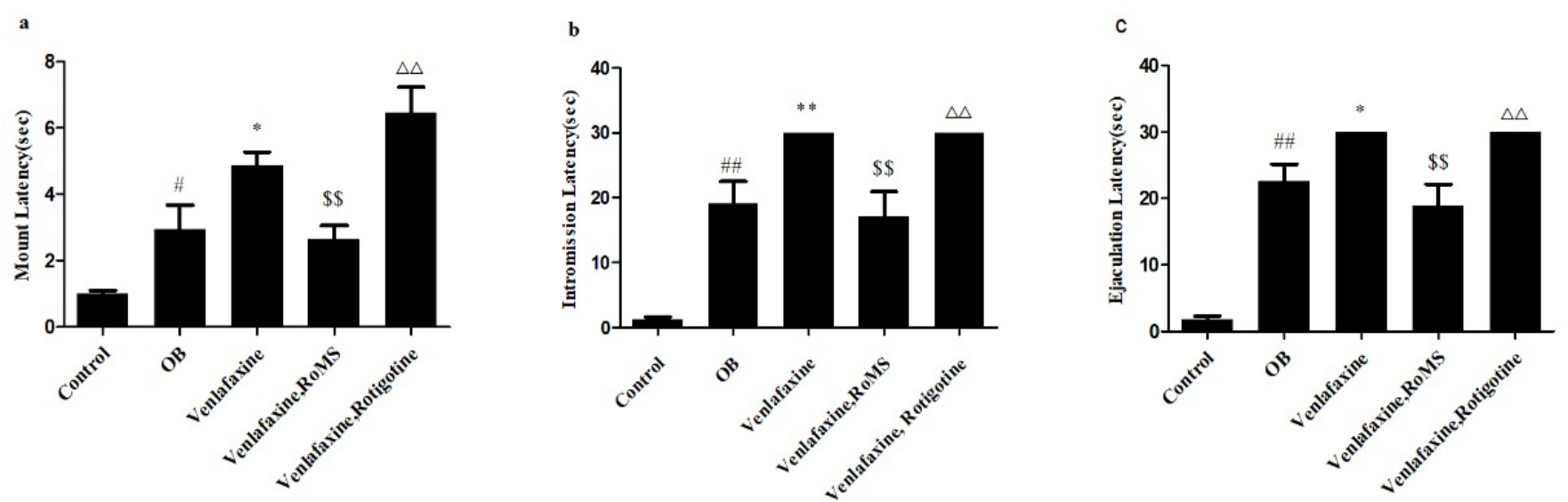

Figure 2: Effects of RoMS on (a) mount latency, (b) intromission latency and (c) ejaculation latency. Data are expressed as mean \pm SEM. ${ }^{*} p<0.05$; ${ }^{\# \#} p<0.01$ when compared to the control group. ${ }^{*} P<0.05 ;{ }^{* *} P<0.01$ when compared to the OB group. ${ }^{\$ \$} p<0.01$ when compared to the venlafaxine group. ${ }^{\Delta \Delta} p<0.01$ when compared to the venlafaxine, RoMS group.

a

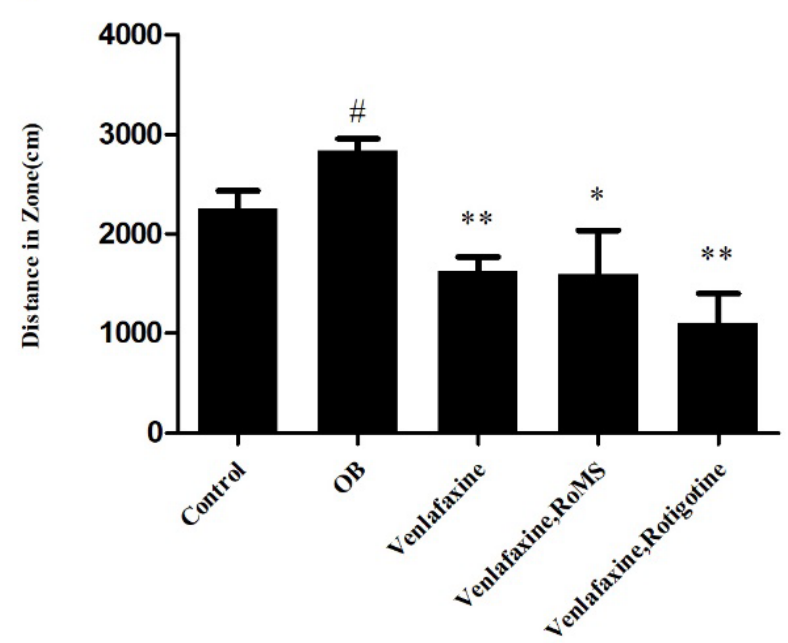

b

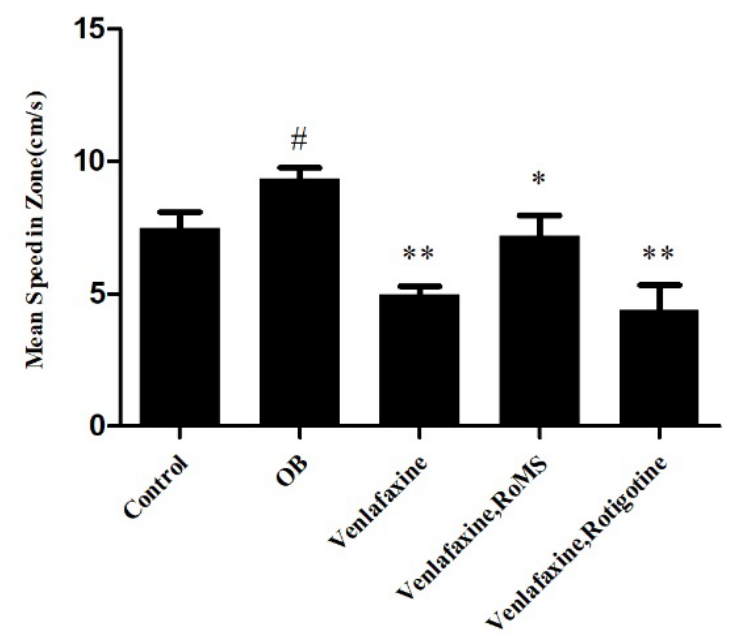

Figure 3: Effects of RoMS on (a) total distance mean speed and (b) mean speed. Data are expressed as mean \pm SEM. ${ }^{*}<<0.05$ when compared to the control group. ${ }^{*}<<0.05 ;{ }^{* *} p<0.01$ when compared to the OB group. 


\section{DISCUSSION}

Bilateral olfactory bulbectomy could induce depressive symptoms respond to chronic antidepressant treatment, thus $\mathrm{OB}$ is considered as one of the best available models to evaluate antidepressant activity [21]. Bulb ablation results in increased hyperactivity in a novel environment [22]. Since the olfactory bulb projects into different regions of the brain, thus ablation of these bulbs results in neurodegeneration in the projection areas, which possibly explains OB-induced copulatory behaviour changes. And venlafaxine can aggravate the OB-induced sexual dysfunction. The results of this study show that the bilateral olfactory bulbectomy increase the hyperactivity of rats in a novel environment. Venlafaxine can decrease the mean speed and the total distance and co-administered venlafaxine with ROMS cannot do better.

Serotonin strongly interacts with noradrenaline and dopamine signalling. SSRIs like fluoxetine may affect sexual behaviour via actions on these monoamines. Preclinical research suggests a promoting role for noradrenaline in the regulation of sexual behaviour. The genital organs are strongly innervated by noradrenergic nerves [23]. Several studies suggest that noradrenaline facilitates ejaculation via activation of $\square 1$-adrenergic receptors in the genital organs, whereas a single report showed that $\square 1$-adrenergic antagonists may delay ejaculation. In addition, noradrenaline levels are increased in the lumbosacral spinal cord during intromission and ejaculation and depletion of central noradrenaline neurons with the neurotoxin DSP4 blocks the stimulatory effects of the non-specific serotonin receptor agonist lisuride. On the basis of the promoting effects of noradrenaline presented in these studies, one could expect that combining serotonin reuptake inhibition with noradrenaline reuptake inhibition could hypothetically counteract the sexual side effects of SSRI. However, human data show that the SNRI venlafaxine, at doses that exert antidepressant efficacy, induces comparable sexual side effects to SSRIs including fluoxetine and paroxetine [24].

\section{CONCLUSION}

The results obtained in this study show that venlafaxine high dose reduced the number of intromission and ejaculation and increased the duration of the latency periods for mount, intromission and ejaculation. These data showing that the sex inhibiting effects of venlafaxine are comparable to those following SSRIs treatment and the RoMS treatment reverse the venlafaxine-induced sexual dysfunction. Together with the comparable sexual side effect profile of venlafaxine, these data suggest that the mechanism underlying sexual side effects of venlafaxine is comparable to that of SSRIs

In conclusion, the present data indicate that RoMS improves the venlafaxine-induced sexual dysfunction and does not affect its antidepressive efficacy.

\section{REFERENCES}

1. Kessler RC, Berglund P, Demler O, Jin R, Koretz D, Merikangas $\mathrm{KR}$, et al. The epidemiology of major depressive disorder: results from the National Comorbidity Survey Replication (NCS-R). JAMA. 2003;289(23):3095-3105.

2. Sartorius N. The economic and social burden of depression. J Clin Psychiatry. 2001;62:8-11.

3. Nestler EJ, Barrot M, DiLeone RJ, Eisch AJ, Gold SJ, Monteggia LM. Neurobiology of depression. Neuron. 2002;34:13-25.
4. Ye L, Hu Z, Du G, Zhang J, Dong Q, Fu F, et al. Antidepressant-like effects of the extract from Cimicifuga foetida L. J Ethnopharmacol. 2012;144:683-691.

5. Hueletl-Soto ME, Carro-Juárez M, Rodríguez-Manzo G. Fluoxetine chronic treatment inhibits male rat sexual behavior by affecting both copulatory behavior and the genital motor pattern of ejaculation. J Sex Med. 2012;9:1015-1026.

6. Segraves RT. Antidepressant-induced sexual dysfunction. J Clin Psychiatry. 2014;121:132-137.

7. Montejo-González AL, Llorca G, Izquierdo JA, Ledesma A, Bousoño M, Calcedo A, et al. SSRI-induced sexual dysfunction: fluoxetine, paroxetine, sertraline, and fluvoxamine in a prospective, multicenter, and descriptive clinical study of 344 patients. J Sex Marital Ther. 1997;23:176-194.

8. Rényi L. The effect of selective 5-hydroxytryptamine uptake inhibitors on 5-methoxy-N,N-dimethyltryptamine-induced ejaculation in the rat. Br J Phar- macol. 1986;87:639-648.

9. Zemishlany Z, Aizenberg D, Weizman A. Subjective effects of MDMA ('Ecstasy') on human sexual function. Eur Psychiatry. 2001;16:127-130.

10. Zajecka J, Fawcett J, Schaff M, Jeffriess H, Guy C. The role of serotonin in sexual dysfunction: fluoxetine-associated orgasm dysfunction. J Clin Psychiatry. 1991;52:66-68.

11. Sorscher SM, Dilsaver SC. Antidepressant-induced sexual dysfunction in men: due to cholinergic blockade? J Clin Psy- chopharmacol. $1986 ; 6: 53-55$.

12. Hull EM, Muschamp JW, Sato S. Dopamine and serotonin: influences on male sexual behavior. Physiology \& behavior. 2004;83:291-307.

13. Bijlsma EY, Chan JS, Olivier B, Veening JG, Millan MJ, Waldinger MD, et al. Sexual side effects of serotonergic antidepressants: mediated by inhibition of serotonin on central dopamine release?. Pharmacol Biochem Behavior.2014;121:88-101.

14. Hull EM, Bazzett TJ, Warner RK, Eaton RC, Thompson JT. Dopamine receptors in the ventral tegmental area modulate male sexual behavior in rats. Brain research. 1990;512:1-6.

15. Mas M, Fumero B, Perez-Rodriguez I. Induction of mating behavior by apomorphine in sexually sated rats. European J pharmacol. 1995;280:331-4

16. Wang A, Wang L, Sun K, Liu W, Sha C, Li Y, et al. Preparation of rotigotine-loaded microspheres and their combination use with L-DOPA to modify dyskinesias in 6-OHDA-lesioned rats. Pharmaceutical Res. 2012;29:2367-2376.

17. Wang A, Liu Y, Liang R, Zhang X, Sun K, Wu Z, et al. Preparation and evaluation of rotigotine-loaded implant for the treatment of Parkinson's disease and its evolution study. Saudi Pharm J 2016;24:363-370.

18. Eisenstein SA, Clapper JR, Holmes PV, Piomelli D, Hohmann AG. A role for 2-arachidonoylglycerol and endocannabinoid signaling in the locomotor response to novelty induced by olfactory bulbectomy. 2010;61:419-429.

19. Zhang XR, Zhang ZJ, Jenkins TA, Cheng WR, Reynolds GP. The dosedependent effect of chronic administration of haloperidol, risperidone, and quetiapine on sexual behavior in the male rat. 2011;8:3345-3353.

20. Li D, Cai Z, Wu J, Zhang Y. Bax inhibitor-1 overexpression in prelimbic cortex protects rats against depression-like behavior induced by olfactory bulbectomy and reduces apoptotic and inflammatory signals. Neurol Res. 2019;41:369-377.

21. Song C, Leonard BE. The olfactory bulbectomised rat as a model of depression. Neurosci Biobehav Rev. 2005;29:627-647. 
22. Hsieh JT, Liu SP, Hsieh CH, Lai MK, Cheng JT. An ex vivo evaluation of regulatory role of biogenic amines in rat seminal vesicle after pharmacological manipulation. Life Sciences. 1998;63:PL221-229.

23. Kepper ME, Keast JR. Location, immunohistochemical features, and spinal connections of autonomic neurons innervating the rat seminal vesicles. Biol Reprod. 1997;57:1164-1174.

24. Bacchiochi JR, Bagby RM, Psych C. Antidepressant-induced sexual dysfunction during treatment with moclobemide, paroxetine, sertraline, and venlafaxine. J Clin Psychiatry. 2000;61. 